Artículo científico

(Original paper)

\title{
PULGONES (HEMIPTERA: APHIDIDAE) Y SUS PARASITOIDES (HYMENOPTERA) EN CULTIVOS DE SORGO EN LOS ESTADOS DE COLIMA Y TAMAULIPAS, MÉXICO
}

\author{
APHIDS (HEMIPTERA: APHIDIDAE) AND ITS PARASITOIDS (HYMENOPTERA) IN \\ SORGHUM CROPS IN THE STATES OF COLIMA AND TAMAULIPAS, MEXICO
}

\begin{abstract}
BEATRIZ RODRÍGUEZ-VÉLEZ ${ }^{1}$, ALBA SUASTE-DZUL ${ }^{2}$, ADRIEN GALLOU ${ }^{3 *}$, JOSÉ MANUEL RODRÍGUEZVÉLEZ ${ }^{4}$, MARIZA ARACELI SARMIENTO-CORDERO ${ }^{5,}$ HUGo CESAR ARREDONDO-BERNAL ${ }^{6}$

Centro Nacional de Referencia de Control Biológico; SENASICA, Carretera Tecomán-Estación FFCC km. 1.5, Col. Tepeyac; C.P. 28110, Tecomán, Colima, México. ${ }^{1}<$ beatriz_rv@ @ yahoo.com>; ${ }^{2}<$ albasuaste@ gmail.com>; ${ }^{3 *}<$ gallou.adrien@ @hotmail.fr $>$;

${ }^{4}<$ jm_rodriguez81@yahoo.com.mx $>;{ }^{5}<$ marizilla@ hotmail.com $>;{ }^{6}<$ hcesar_64@yahoo.com.mx $>$

* Autor de correspondencia: <gallou.adrien@hotmail.fr>
\end{abstract}

Recibido: 20/09/2018; aceptado: 13/12/2018; publicado en línea: 31/01/2019

Editor responsable: Magdalena Cruz

Rodríguez-Vélez, B., Suaste-Dzul, A., Gallou, A., Rodríguez-Vélez, J. M., Sarmiento-Cordero, M. A., Arredondo-Bernal, H. C. (2019) Pulgones (Hemiptera: Aphididae) y sus parasitoides (Hymenoptera) en cultivos de sorgo en los estados de Colima y Tamaulipas, México. Acta Zoológica Mexicana (nueva serie), 35, 1-9. https://doi.org/10.21829/azm.2019.3501085

RESUMEN. El pulgón amarillo del sorgo Melanaphis sacchari y el verde Schizaphis graminum son especies que coexisten en cultivos de sorgo. El pulgón verde se reportó por vez primera para México en 1972 en cultivos de avena, y el amarillo en 2013 en cultivo de sorgo, este último causando pérdidas hasta del $100 \%$. Desde entonces, se realizan esfuerzos para su control, por ello, el objetivo de este estudio fue conocer los parasitoides de M. sacchari y $S$. graminum en cultivos de sorgo en Colima y Tamaulipas. En 2014 se realizaron muestreos en cinco localidades de Tamaulipas afectadas por el pulgón amarillo, donde se colectaron pulgones parasitados. La recolecta en Colima fue en 2016, realizándose semanalmente durante un ciclo completo del sorgo con red de barrido y colecta directa. Se registraron dos especies de parasitoides: Lysiphlebus testaceipes, donde se reconocieron a nivel molecular dos haplotipos, uno por estado, 15 especímenes en Tamaulipas emergiendo del pulgón amarillo y seis en Colima también emergiendo del pulgón amarillo y 127 recolectados con red en Colima y, Pachyneuron aphidis hiperparasitoide de $L$. testaceipes, se registraron tres individuos emergiendo de momias de $M$. sacchari en Tamaulipas y dos en Colima, además de 12 especímenes recolectados con red en este estado. En Colima, el pulgón verde y $L$. testaceipes mostraron mayor presencia durante el desarrollo de las hojas e iniciación de la panoja, mientras que el pulgón amarillo fue más abundante durante el desarrollo y llenado de los granos, durante esa etapa la presencia de parasitoides fue casi nula. Concluyendo que L. testaceipes y $P$. aphidis aparentemente muestran preferencia por $S$. graminum en el área de estudio.

Palabras clave: Melanaphis sacchari; Schizaphis graminun; Lysiphlebus testaceipes; Pachyneuron aphidis 
Rodríguez-Vélez, B., Suaste-Dzul, A., Gallou, A., Rodríguez-Vélez, J. M., Sarmiento-Cordero, M. A., Arredondo-Bernal, H. C. (2019) Aphids (Hemiptera: Aphididae) and its parasitoids (Hymenoptera) in Sorghum crops in the states of Colima and Tamaulipas, Mexico. Acta Zoológica Mexicana (nueva serie), 35, 1-9. https://doi.org/10.21829/azm.2019.3501085

\begin{abstract}
The sugarcane aphid Melanaphis sacchari and the green aphid Schizaphis graminum, are species that coexist in sorghum crops. The green aphid was reported for Mexico in 1972 in oats and the sugarcane aphid in 2013 in sorghum, causing the last one loss up to 100\%. Since then, efforts are underway for their control; therefore, the objective of this study was to find parasitoids associated to M. sacchari and $S$. graminum in sorghum crops in Colima and Tamaulipas. In Tamaulipas, five localities affected by the sugarcane aphid were sampled in 2014 by collecting parasitized aphids. In Colima the collecting was weekly during a cycle of sorghum, by netting and direct collection, in 2016. Two species of parasitoids were recorded, Lysiphlebus testaceipes where two haplotypes were recognized, one for each state, and Pachyneuron aphidis, hyperparasitoid of L. testaceipes. The green aphid and L. testaceipes showed greater presence during the development of the leaves and beginning of blooming, while the sugarcane aphid was more abundant during the development and filling of the grains, during that stage in the case of sugarcane aphid, the presence of parasitoids was almost null. Concluding that L. testaceipes and P. aphidis show preference for $S$. graminum in the study area.
\end{abstract}

Key words: Melanaphis sacchari; Schizaphis graminun; Lysiphlebus testaceipes; Pachyneuron aphidis

\title{
INTRODUCCIÓN
}

El pulgón amarillo del sorgo, Melanaphis sacchari (Zehntner 1897) y el pulgón verde de los cereales, Schizaphis graminum (Rondani 1852), son especies comunes en el cultivo de sorgo. El pulgón verde de los cereales está ampliamente distribuido en Asia, África, Australia y América, convirtiéndose en una plaga de importancia económica. En México se registró por primera vez en 1972 (Peña \& Sifuentes, 1972), siendo sus hospederos preferidos la avena, cebada, centeno, trigo, sorgo y otras gramíneas silvestres (Shufran, 2011). Así mismo, el pulgón amarillo del sorgo se reportó para México en 2013, en el estado de Tamaulipas, donde atacó principalmente cultivos de sorgo y causó pérdidas hasta del 100\% (Maya-Hernández \& Rodríguez-del-Bosque, 2014). Lo anterior propició el desarrollo de estudios relacionados para el control de estas plagas; por lo que el propósito de este trabajo fue conocer las especies de parasitoides asociadas a los pulgones verde y amarillo del sorgo en plantaciones de sorgo en los estados de Tamaulipas y Colima. En el estado de Tamaulipas, el estudio se limitó a conocer la identidad de los pulgones y sus parasitoides, mientras que, en Colima, además de la identificación de los diferentes pulgones y sus parasitoides se estudió la fluctuación de la abundancia de estas especies a través de un ciclo completo del sorgo.

\section{MATERIALES Y MÉTODOS}

Área de estudio. El trabajo de campo se realizó en cultivos de sorgo en los estados de Tamaulipas y Colima. Las recolectas en el estado de Tamaulipas se llevaron a cabo durante abril de 2014 en cinco localidades (una colecta por localidad), todas ellas siniestradas por M. sacchari (Cuadro 1).

En el estado de Colima las recolectas se realizaron en la población de Tecuanillo, en el municipio de Tecomán, Colima, México, con coordenadas de $18^{\circ} 51^{\prime} 31^{\prime \prime} \mathrm{N}, 103^{\circ} 52^{\prime} 55^{\prime \prime} \mathrm{O}$, a $17 \mathrm{msnm}$. El predio muestreado consta de 4 ha cultivadas con sorgo (Sorghum bicolor [L.] Moench, SG PICHON 70), cuyo terreno colinda al norte y sur con cultivos de coco (Cocos nucifera L.), al este con un arroyo de temporal, previo a la carretera, y al oeste con vegetación de bosque tropical caducifolio perturbado. 
Cuadro 1. Localidades donde se llevaron a cabo recolectas de pulgones momificados (M. sacchari) en el estado de Tamaulipas, y los parasitoides emergidos.

\begin{tabular}{llllll}
\hline Ciudad & Ejido & Georeferencias & $\begin{array}{l}\text { Altitud } \\
(\mathrm{msnm})\end{array}$ & $\begin{array}{l}\text { Lysiphlebus } \\
\text { testaceipes }\end{array}$ & $\begin{array}{l}\text { Pachyneuron } \\
\text { aphidis }\end{array}$ \\
\hline Tampico & Buenos Aires & $\begin{array}{l}22^{\circ} 26^{\prime} 15^{\prime \prime} \mathrm{N} \\
98^{\circ} 01^{\prime} 28^{\prime \prime} \mathrm{O}\end{array}$ & 11 & 5 machos & 2 hembras \\
Altamira & Cervantes & $\begin{array}{l}12^{\circ} 26^{\prime} 29^{\prime \prime} \mathrm{N} \\
98^{\circ} 02^{\prime} 08^{\prime \prime} \mathrm{O}\end{array}$ & 8.5 & 1 macho & 1 macho \\
Altamira & Cervantes & $\begin{array}{l}22^{\circ} 26^{\prime} 54^{\prime \prime} \mathrm{N} \\
98^{\circ} 03^{\prime} 50^{\prime \prime} \mathrm{O}\end{array}$ & 19.2 & 3 machos & \\
Altamira & Esteros & $\begin{array}{l}22^{\circ} 30^{\prime} 39^{\prime \prime} \mathrm{N} \\
98^{\circ} 06^{\prime} 58^{\prime \prime} \mathrm{O}\end{array}$ & 14.69 & 3 machos & \\
Altamira & Villa Cuauhtémoc & $22^{\circ} 31^{\prime} 58^{\prime \prime} \mathrm{N}$ & 59 & 3 machos & \\
& & $98^{\circ} 09^{\prime} 40^{\prime \prime} \mathrm{O}$ & & & \\
\hline
\end{tabular}

Método de muestreo y procesamiento de muestras. Tamaulipas: La recolecta se realizó examinando hojas de las plantas infectadas con pulgones, colectando aquellos pulgones que presentaron diferente aspecto al resto, como estar inmóviles o de diferente coloración. Los pulgones seleccionados fueron colocados en viales de plástico de $20 \mathrm{ml}$ con tapa a presión, posteriormente, se trasladaron al laboratorio donde se mantuvieron en cajas Petri de plástico de $5.5 \mathrm{~cm}$ de diámetro, bajo condiciones de $26^{\circ} \mathrm{C}$ y $65 \% \mathrm{HR}$, hasta la emergencia de los parasitoides. Colima: Las recolectas se realizaron semanalmente a lo largo del ciclo del cultivo (febrero-julio 2016) con red de barrido y recolecta directa de pulgones momificados o con signos de parasitismo. Con la red de barrido se muestrearon tres surcos al azar, equivalentes a $150 \mathrm{~m}$; los insectos recolectados se colocaron en bolsas plásticas con alcohol etílico al $70 \%$ como líquido conservador, posteriormente las muestras fueron llevadas al laboratorio para ser procesadas. Los pulgones momificados recolectados manualmente fueron colocados individualmente en cajas Petri de plástico de $5.5 \mathrm{~cm}$ de diámetro, que se mantuvieron en laboratorio bajo condiciones de $26^{\circ} \mathrm{C}$ y $65 \%$ HR en espera de la emergencia de los parasitoides.

El trabajo de laboratorio incluyó el procesamiento de 21 muestras obtenidas con red de barrido del estado de Colima. Los pulgones y parasitoides fueron separados, los parasitoides fueron secados, montados y etiquetados, mientras que los pulgones se mantuvieron en alcohol etílico al $70 \%$.

Identificación morfológica de pulgones y parasitoides. La identificación morfológica de los parasitoides se obtuvo con apoyo de literatura especializada (Hui et al., 2009; Havelka et al., 2012), al igual que la identificación de los pulgones (Hayder \& Nassreen, 2012). Los pulgones y parasitoides recolectados se depositaron en la Colección de Insectos Entomófagos del Centro Nacional de Referencia de Control Biológico (CIE-CNRCB), ubicado en Tecomán, Colima, México.

Identificación molecular de los parasitoides. El ADN de los parasitoides se obtuvo con la técnica HotSHOT (Truett et al., 2000), la cual fue modificada para la recuperación íntegra del exoesqueleto de los ejemplares como un protocolo no destructivo del tejido (Guzmán-Larralde et al., 2017). Para la identificación molecular, se amplificó la región barcoding del Citocromo Oxidasa subunidad I (COI) en termociclador C1000 ${ }^{\mathrm{TM}}$ (Bio-Rad Laboratories, Inc.). Los primers universales para la amplificación y secuenciación fueron: LepF1/LepR1, que amplifican un fragmento de $658 \mathrm{pb}$ del ADN mitocondrial (Hajibabaei et al., 2006). La mezcla de reactivos para LepF1/LepR1 se desarrolló en volúmenes de $20 \mu 1$, los cuales contenían $0.5 \mu \mathrm{m}$ de cada primer, $1 \mathrm{X}$ buffer Phire reaction (5X) (Thermo Fisher Scientific $\odot$, MA, USA) que contiene $1.5 \mathrm{~mm}$ de $\mathrm{MgCl}_{2}$ en la concentración final, $0.2 \mathrm{~mm}$ de cada dNTP (Promega $\odot$, WI, USA), $0.4 \mu 1$ de Phire Hot Start II Polimerasa (Thermo Scientific Inc., MA, USA) y $2 \mu 1$ de ADN genómico. 
Las secuencias COI se editaron con el programa BioEdit Sequencer (Hall, 1999) y se alinearon con el algoritmo MUSCLE (Edgar, 2004) en Mega 7 (Kumar et al., 2016). Las distancias genéticas fueron calculadas empleando el modelo Kimura 2-parámetros para los insectos bajo estudio (Kimura, 1980) y la secuencia de Lysiphlebus fabarum (Marshall) (Accesión: KP663457). Las secuencias consenso se compararon en las bases de datos de ácidos nucleicos GenBank y Barcode of Life Data Systems (BOLD) (Ratnasingham \& Hebert, 2007) para la identificación. Las secuencias del fragmento COI de cada espécimen de parasitoide fueron depositadas en BOLD y GenBank.

Abundancia. Se realizó un conteo de los pulgones y parasitoides recolectados semanalmente con la red de barrido en Colima, además se cuantificaron los pulgones momificados y el número de parasitoides emergidos de éstos, datos que se analizaron comparando el número de insectos a través del desarrollo del cultivo.

\section{RESULTADOS}

Las avispas Lysiphlebus testaceipes (Cresson, 1880) (Ichneumonoidea: Braconidae) y Pachyneuron aphidis (Bouché, 1834) (Chalcidoidea: Pteromalidae) se registraron en Tamaulipas, emergiendo de pulgones momificados de M. sacchari. En cambio, en Colima se les registró a ambos parasitoides asociados a ambos áfidos M. sacchari y S. graminum.

Los productos de PCR para la región COI fueron amplificados y secuenciados exitosamente para las muestras de L. testaceipes de Colima ( ${ }^{\circ}$ Bold: AGAAA001-17; GenBank: KY754678) y Tamaulipas ( $\mathrm{N}^{\circ}$ Bold: AGAAA003-17; GenBank: KY754677). Ambos obtuvieron altos porcentajes de identidad (99.68-100\%) consistentes con la especie L. testaceipes sometida en GenBank y Bold Systems. Las secuencias de ADN de L. testaceipes de Colima y Tamaulipas mostraron haplotipos diferentes para cada estado. Las distancias genéticas obtenidas entre ambas muestras de L. testaceipes y del grupo externo $(L$. fabarum [Marshall, 1896]) se encuentran entre 7.9-7.5\%; esto indica que los especímenes de Colima y Tamaulipas pertenecen a una sola especie (L. testaceipes) con tres sitios de cambios nucleotídicos en la región COI, definiéndose así dos haplotipos de la especie (Cuadro 2).

Cuadro 2. Posiciones en las secuencias de ADN de Lysiphlebus testaceipes con cambios nucleotídicos.

\begin{tabular}{llll}
\hline Especímenes & \multicolumn{3}{c}{ Sitios polimórficos de la región mitocondrial COI $\left(5^{\prime}-3^{\prime}\right)$} \\
\hline & $303 \mathrm{pb}$ & $336 \mathrm{pb}$ & $450 \mathrm{pb}$ \\
Colima & $\mathrm{G}$ & $\mathrm{A}$ & $\mathrm{C}$ \\
Tamaulipas & $\mathrm{A}$ & $\mathrm{G}$ & $\mathrm{T}$ \\
\hline
\end{tabular}

A: Adenina; T: Timina; C: Citosina; G: Guanina.

Abundancia. En Tamaulipas se recolectó un total de 32 pulgones amarillos del sorgo parasitados, de los cuales, emergieron 18 individuos: 15 L. testaceipes y tres de $P$. aphidis (Cuadro 1). Durante las colectas no se registró la presencia del pulgón verde.

En cambio, en Colima se recolectó un total de 27,454 especímenes de M. sacchari y 611 especímenes de $S$. graminum con red de barrido (Fig. 1); además de 86 parasitoides de L. testaceipes y 12 de $P$. aphidis (Fig. 2). 


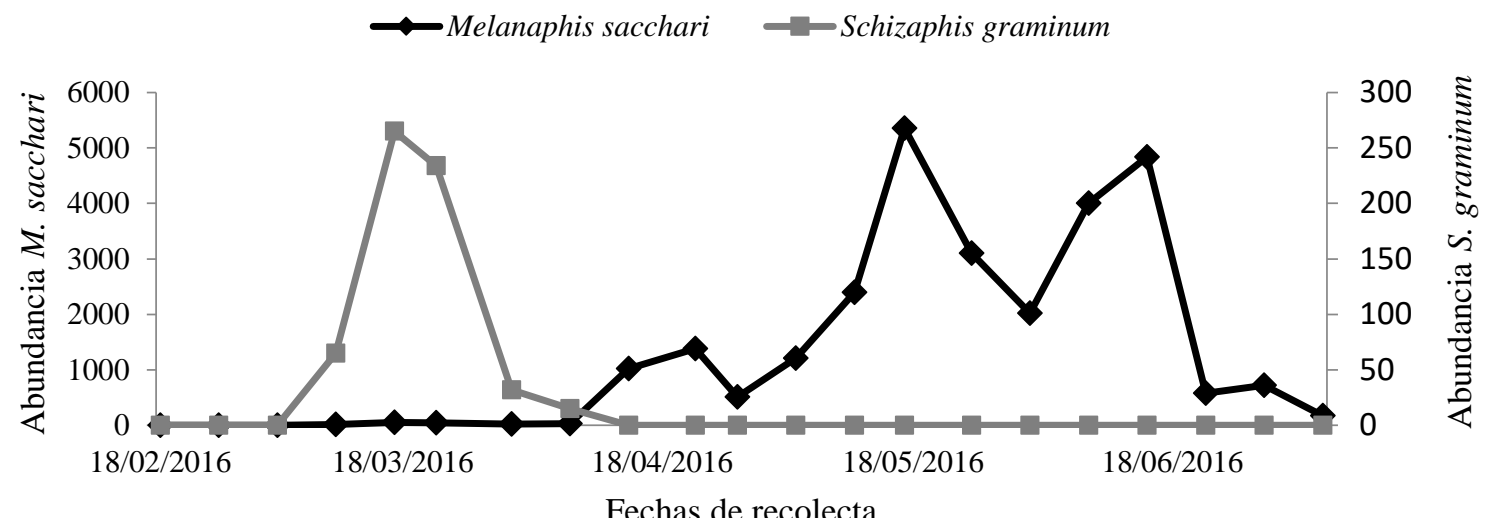

Figura 1. Número de pulgones recolectados con red de barrido durante el ciclo primavera-verano del sorgo en Tecomán, Colima.

En Colima, en el mes de marzo se recolectó un total de 206 áfidos momificados (187 de $S$. graminum y 19 de $M$. sacchari), posteriormente no se observaron áfidos parasitados en campo; de éstos, sólo en 150 se observó la emergencia de parasitoides. De las momias de $S$. graminum emergieron 127 avispas pertinentes a L. testaceipes y 15 a P. aphidis; mientras que de las momias de $M$. sacchari emergieron seis especímenes de L. testaceipes y dos de P. aphidis.

La especie $S$. graminum se observó en el estado de Colima durante el mes de marzo y la primera semana de abril (periodo de crecimiento de las hojas e inició el desarrollo de la panoja), que corresponde con la mayor abundancia de L. testaceipes (Figs. 1-2). Posteriormente la presencia del pulgón verde fue nula y la del bracónido mínima. El hiperparasitoide $P$. aphidis fue registrado del 17 de marzo hasta la última semana de mayo (Fig. 2).

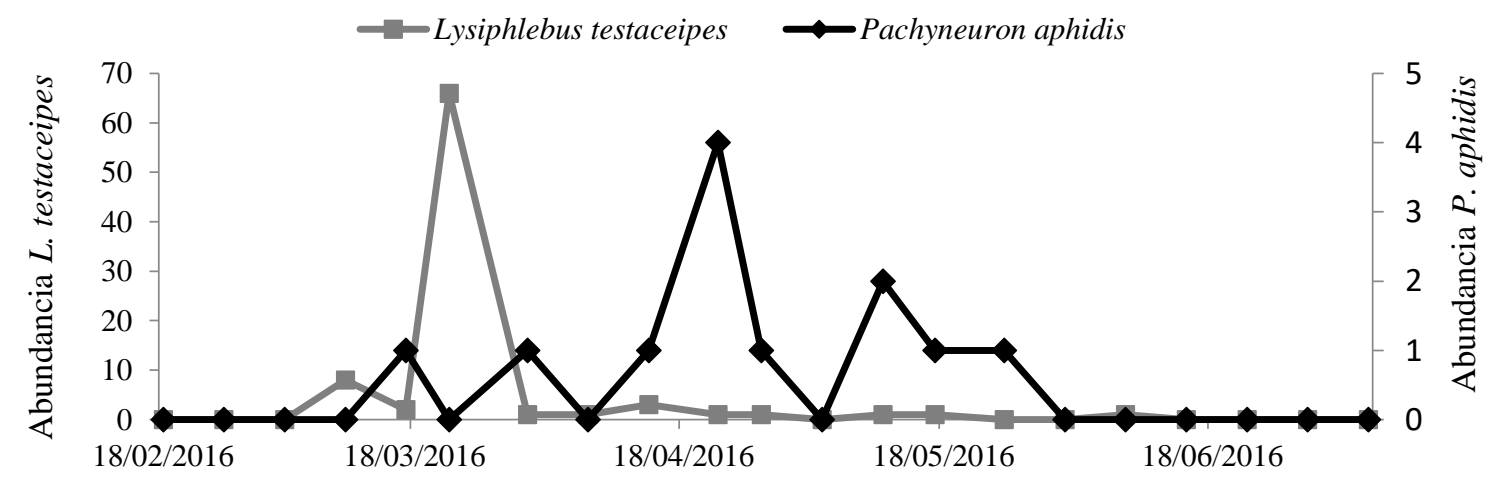

Fechas de recolecta

Figura 2. Número de avispas parasitoides recolectadas con red de barrido durante el ciclo primaveraverano del sorgo en Tecomán, Colima.

La densidad poblacional de $M$. sacchari en Colima mostró su máxima abundancia durante los meses de mayo y junio (Fig. 1), que corresponde a la etapa del desarrollo y llenado de los granos de sorgo. Sin embargo, durante ese periodo ya no hubo registros del pulgón verde y la presencia de parasitoides fue baja. El parasitoide L. testaceipes recolectado con red de barrido tuvo mayor presencia durante el mes de marzo, 
al igual que los emergidos de los pulgones momificados, mostrando su máxima abundancia durante la iniciación de la panoja. Los especímenes de $P$. aphidis fueron en general poco abundantes en comparación con L. testaceipes, teniendo mayor presencia durante el desarrollo de la inflorescencia.

\section{DISCUSIÓN}

Pachyneuron aphidis es un hiperparasitoide de la subfamilia Aphidiinae (Braconidae) (Zamora-Mejías \& Hanson, 2016); en este estudio se le registró parasitando a L. testaceipes (parasitoide primario de pulgones), actúa como ectoparasitoide idiobionte cuando éste parasita a pulgones (Bruner et al., 1975).

La especie L. testaceipes es un endoparasitoide koinobionte y solitario de ninfas y adultos de pulgones (Vázquez et al., 2008); esta avispa ha sido reportada como parasitoide primario de ambos pulgones, verde y amarillo. Bowling et al. (2016) mencionan el parasitismo de este bracónido sobre pulgón amarillo en Estados Unidos de Norte América, sin embargo, registraron pocos especímenes de L. testaceipes emergiendo de los pulgones momificados en comparación con una especie de la familia Aphelinidae, Aphelinus sp., grupo varipes. Colares et al. (2015) reportaron para Kansas, Estados Unidos a Aphelinus sp. parasitando a ambos pulgones; mientras que, L. testaceipes parasitó exclusivamente al pulgón verde; durante ese estudio se observó que dicho parasitoide no logró desarrollarse en $M$. sacchari, debido a que el pulgón amarillo estaba infectado con el endosimbionte Hamiltonella defensa, bacteria que protege a los pulgones del parasitismo de avispas de la subfamilia Aphiidinae (Vorburger et al., 2009).

En el caso de México, Rodríguez-del-Bosque y Terán (2015) señalan una especie de la familia Braconidae y otra de Aphelinidae parasitando a M. sacchari, sin mencionar la identidad específica de éstas. Por otro lado, Vázquez-Navarro et al. (2016) registran la presencia de Lysiphlebus sp., parasitando al pulgón amarillo en sorgo en el estado de Durango, al igual que López-Gutiérrez et al. (2016) lo reportan en el estado de Guanajuato. En cambio, en Michoacán registran a L. testaceipes parasitando al pulgón verde en cultivos de trigo (Bahena-Juárez et al., 2012).

Lysiphlebus testaceipes es considerado como agente de control biológico de pulgones, especialmente de S. graminum, incluso está disponible a nivel comercial (Beyl \& Trigiano, 2014); por ejemplo en España es producido por las empresas Iberfol S.L., Mip System Agro S.L., Técnicas de Control Biológico S.L., por lo que se consideró importante el uso de técnicas moleculares para conocer la diversidad genética de los especímenes recolectados; en este caso se reconocieron dos haplotipos, estas técnicas son significativas para establecer estrategias para el desarrollo de programas de control biológico, ya que permiten la identificación de biotipos endocriados y silvestres (Aljanabi et al., 1998).

En la recolecta en el estado de Tamaulipas no fue posible detectar al pulgón verde, posiblemente porque fue un periodo corto (una semana) y a la fase en que estaba el cultivo durante la recolecta, que estaba alrededor de dos meses de la siembra y en muy malas condiciones por la presencia de $M$. sacchari, no obstante que $S$. graminum es una especie común en cultivos de sorgo en Tamaulipas (Rodríguez-del-Bosque \& Loera, 2006).

En Colima, se registró un menor número de pulgones amarillos del sorgo parasitados por $L$. testaceipes, en contraste con el parasitismo en pulgón verde, lo que fue similar al presentado por Bowling et al. (2016), al registrar una escasa emergencia de L. testaceipes de $M$. sacchari, inclusive las avispas emergidas, presentaban un color más obscuro y una apariencia más bulbosa a la conocida para este bracónido; no obstante, en el presente estudio no se observó alguna apariencia diferente de los especímenes de L. testaceipes. 
Las poblaciones de L. testaceipes y del pulgón verde $S$. graminum recolectados en Colima mostraron patrones muy similares de densidad (Figs. 1-2), lo que sugiere que este bracónido ataca con mayor frecuencia al pulgón verde en la zona de estudio. El ataque de L. testaceipes a S. graminun ha sido registrado frecuentemente (Giles et al., 2003; Jones et al., 2007), ya que se ha observado que las poblaciones de $S$. graminum se pueden mantener por debajo del umbral económico de daño en cultivos de trigo, gracias al parasitismo ejercido por este parasitoide (Giles et al., 2003).

\section{CONCLUSIONES}

Las avispas L. testaceipes y P. aphidis se presentan en Tamaulipas asociadas al pulgón M. sacchari. Así mismo, en Colima se les registró asociadas con los pulgones $M$. sacchari y $S$. graminum. Se registran dos haplotipos para L. testaceipes, uno para Tamaulipas y otro para Colima. Del total de parasitoides que emergieron de pulgones momificados en Colima, sólo el 5.3\% emergieron del pulgón amarillo del sorgo. De acuerdo con las muestras obtenidas con red de barrido y la colecta de pulgones momificados, aparentemente los parasitoides L. testaceipes y P. aphidis se presentan con mayor frecuencia sobre el pulgón verde.

\section{LITERATURA CITADA}

Aljanabi, S. M., Loiácono, M. S., Loruenço, R. T., Borges, M., Tigano, M. S. (1998) RAPD analysis revealing polymorphism in egg parasitoids of soybean stink bugs (Hemiptera: Pentatomidae). Anais da Sociedade Entomologica do Brasil, 27 (3), 413-420.

Bahena-Juárez, F., Velázquez-García, J. de J., Báez-Pérez, A. (2012) Manejo agroecológico de plagas en trigo para una agricultura de conservación en el Valle Morelia-Querendaro, Michoacán. Instituto Nacional de Investigaciones Forestales Agrícolas y Pecuarias, Centro de Investigación Regional Pacífico Centro. Campo experimental Uruapan, Folleto Técnico Núm. 30, Uruapan, Michoacán, México. Disponible en: http://biblioteca.inifap.gob.mx:8080/jspui/handle/123456789/3533 (acceso en enero de 2017).

Beyl, C. A., Trigiano, R. N. (2014) Plant propagation concepts and laboratory exercises. CRC press, 479 pp.

Bowling, R. D., Brewer, M. J., Kerns, D. L., Gordy, J., Seiter, N., Elliot, N. E., Buntin, G. D., Way, M. O., Royer, T. A., Biles, S., Maxson, E. (2016) Sugarcane aphid (Hemiptera: Aphididae): A new pest on sorghum in North America. Journal of Integrated Pest Management, 7 (1), 1-13.

Bruner, S. C., Scaramuzza, L. C., Otero, A. R. (1975) Catálogo de los insectos que atacan a las plantas económicas de Cuba-2. Academia de Ciencias de Cuba. Instituto de Zoología, Habana, Cuba, 401 pp.

Colares, F., Michaud, J. P., Clint, L. B., Torres, J. B. (2015) Recruitment of aphidophagous arthropods to sorghum plants infested with Melanaphis sacchari and Schizaphis graminum (Hemiptera: Aphididae). Biological Contol, 90, 16-24.

Edgar, R. C. (2004) MUSCLE: a multiple sequence alignment method with reduced time and space complexity. BMC Bioinformatics, 5, 1-19.

Giles, K. L., Jones, D. B., Royer, T. A., Elliott, N. C., Kindler, S. D. (2003) Development of a sampling plan in winter wheat that estimates cereal aphid parasitism levels and predicts population suppression. Journal of Economic Entomology, 96, 975-982.

Guzmán-Larralde, A. J., Suaste-Dzul, A. P., Gallou, A., Peña-Carrillo, K. I. (2017) DNA recovery from microhymenoptera using six non-destructive methodologies with considerations for subsequent preparation of museum slides. Genome, 60, 85-91. 
Hajibabaei, M., Janzen, D. H., Burns, J. M., Hallwachs, W., Hebert, P. D. N. (2006) DNA barcodes distinguish species of tropical Lepidoptera. Proceeding of the National Academy of Science of the United States of America-PNAS, 103 (4), 968-971.

Hall, T. A. (1999) BioEdit: A user-friendly Biological Sequence Alignment Editor and Analysis Program for Windows 95/98/NT. Nucleic Acid Symposium Series, 41, 95-98.

Havelka, J., Tomanovič, Ž., Kavallieratos, N. G., Rakhshani, E., Pons, X., Petrović, A., Pike, K. S., Starý, P. (2012) Review and key to the world parasitoids (Hymenoptera: Braconidae: Aphidiinae) of Aphis ruborum (Hemiptera: Aphididae) and its role as a host reservoir. Annals of the Entomological Society of America, 105, 386-394.

Hayder, B. A., Nassreen, N. M. (2012) Pictorial key to apterous aphids species (Homoptera: Aphididae, Aphidinae) infested grasses (Gramineae) from several provinces of Iraq. Al-Mustansiriyah Journal of Science, 23 (7), 57-74.

Hui, X., Tian-Yang, J., Da-Wei, H. (2009) Pachyneuron (Hymenoptera: Pteromalidae) from China. Oriental Insects, 43, 341-359.

Jones, D. B., Kristopher, L., Giles, K. L., Elliot, N. C., Payton, M. E. (2007) Parasitism of greenbug, Schizaphis graminum, by the parasitoid Lysiphlebus testaceipes at winter temperatures. Environmental Entomology, 36 (1), 1-8.

Kimura, M. (1980) A simple method for estimating evolutionary rate of base substitutions through comparative studies of nucleotide sequences. Journal of Molecular Evolution, 16, 111-120.

Kumar, S., Stecher, G., Tamura, K. (2016) MEGA7: Molecular Evolutionary Genetics Analysis Version 7.0 for Bigger Datasets. Molecular Evolutionary Genetics Analysis, 33 (7), 1870-1874.

López-Gutiérrez, D. R., Salas-Araiza, M. D., Martínez-Jaime, O. A., Salazar-Solis, E. (2016) Géneros de Aphidiidae (Hymenoptera) parasitando al pulgón amarillo de la caña de azúcar Melanaphis sacchari Zehntner, 1897 (Hemiptera: Aphididae) en Irapuato, Guanajuato, México. Entomología mexicana, 3, 365-368.

Maya-Hernández., A., Rodríguez-del-Bosque, L. (2014) Pulgón Amarillo Melanaphis sacchari, nueva plaga del sorgo en Tamaulipas. Disponible http://www.sagarpa.gob.mx/Delegaciones/tamaulipas/Documents/Pulgon\%20amarillo.pdf (acceso en marzo de 2017).

Peña, R., Sifuentes, J. A. (1972) Lista de cultivos y sus principales plagas en México. Agricultura Técnica en México, 3 (5), 178-193.

Ratnasingham, S., Hebert, P. D. N. (2007) BOLD: The Barcode of Life Data System (www.barcodinglife.org). Molecular Ecology Notes, 7, 355-364.

Rodríguez -del-Bosque, L. A., Terán, A. (2015) Melanaphis sacchari (Hemiptera: Aphididae). A new sorghum insect pest in México. Southwestern Entomologist, 40, 433-434.

Rodríguez-del-Bosque, L. A., Loera, J. (2006) Entomología, pp. 163-191. In Rodríguez-del-Bosque, L. A. (Ed.), 50 Años de Investigación Agropecuaria en el Norte de Tamaulipas, Historia, Logros y Retos. Río Bravo, Tamaulipas, México.

Shufran, K. A. (2011) Host race evolution in Schizaphis graminum (Hemiptera: Aphididae): nuclear DNA sequences. Environmental Entomology, 40 (5), 1317-1322.

Truett, G. E., Heeger, P., Mynatt, R. L., Truett, A. A., Walker, J. A., Warman, M. L. (2000) Preparation of PCR-quality mouse genomic DNA with hot sodium hydroxide and tris (HotSHOT). Biotechniques, 29, 52-54.

Vázquez, L. L., Matienzo, Y., Veitía, M., Alfonso, J. (2008) Conservación y manejo de enemigos naturales de insectos fitófagos en los sistemas agrícolas de Cuba. Instituto de Investigaciones de Sanidad Vegetal, Centro de Información y Documentación de Sanidad Vegetal (IDISAV), La Habana, Cuba, 202 pp.

Vázquez-Navarro, J. M., Carrillo-Aguilera, J. C., Cisneros-Flores, B. A. (2016) Estudio poblacional en un cultivar de sorgo forrajero infestado con pulgón amarillo del sorgo Melanaphis sacchari (Zehntner, 1897) (Hemiptera: Aphididae) en la comarca lagunera. Entomología mexicana, 3, 395400 . 
Vorburger, C., Sandrock, C., Gouskov, A., Castaneda, L .E., Ferrari, J. (2009) Genotypic variation and the role of defensive endosymbionts in an all-parthenogenetic host-parasitoid interaction. International Journal of Organic Evolution, 63, 1439-1450.

Zamora-Mejías, D., Hanson, P. (2016) Trophic relationships of aphid hyperparasitoids (Hymenoptera) in Costa Rica. Journal of the Entomological Research Society, 18 (3), 35-44. 